\title{
Myeloperoxidase Gene Variation as a Determinant of Atherosclerosis Progression in the Abdominal and Thoracic Aorta: An Autopsy Study
}

\author{
Riikka Mäkelä, Pekka J. Karhunen, Tarja A. Kunnas, Erkki Ilveskoski, Olli A. Kajander, \\ Jussi Mikkelsson, Markus Perola, Antti Penttilä, and Terho Lehtimäki \\ Laboratory of Atherosclerosis Genetics (RM, PJK, El, TL), Department of Clinical Chemistry, Center for Laboratory \\ Medicine, Tampere University Hospital, and Department of Forensic Medicine (PJK, TAK, El, OAK, JM), University \\ of Tampere Medical School, Tampere, Department of Forensic Medicine (AP), University of Helsinki, Finland; and \\ Department of Human Genetics (MP), University of California, Los Angeles, California
}

\begin{abstract}
SUMMARY: Myeloperoxidase (MPO) is an enzyme that transforms low-density lipoprotein into atherogenic particles. The MPO gene has a promoter polymorphism at position -463 , which affects gene transcription and leads to high- (G/G) and low-expression (A/A, A/G) genotypes. To determine if these genotypes are associated with the severity of atherosclerosis, we performed an autopsy study of 300 men aged 33 to 69 years (Helsinki Sudden Death Study). We examined the percentage area of fatty streaks and fibrotic, calcified, and complicated lesions using computer-assisted planimetry. The MPO genotypes were determined by PCR. There were significant interactions of MPO genotype with the mean area of fibrotic $(p<0.01)$ and calcified $(p<0.05)$ lesions in the abdominal aorta and in fibrotic lesions in the thoracic aorta $(p=0.003)$. In the abdominal aorta, men $<$ 53 years with low-expression genotypes had on average a $38.6 \%$ larger area of fibrotic lesions and a $43.8 \%$ larger area of calcified lesions than did the subjects with the $\mathrm{G} / \mathrm{G}$ genotype. This association weakened with advancing age. Among men $<53$ years, the MPO genotype was an independent predictor of fibrotic $(p=0.037)$ and calcified $(p=0.001)$ lesion area in the abdominal aorta after adjustment for age, body mass index, diabetes, hypertension, and smoking. MPO gene variation may modify the extent of advanced atherosclerotic lesions in the human aorta in early middle age. (Lab Invest 2003, 83:919-925).
\end{abstract}

therosclerosis is characterized by an inflamma-
tory component as evidenced by the presence of
cells of the immune system, such as phagocytes,
while the atherosclerotic lesion develops (Berliner et
al, 1995; Ross, 1999). Once activated, the phagocytic
cells generate reactive oxygen species that induce
destructive oxidizing stress to the vessel wall (Babior,
2000). In atherosclerosis, the chronic inflammation of
the artery wall leads to an exaggerated response of
the phagocytic cells, which results in oxidation of
low-density lipoprotein (LDL). LDL particles become
atherogenic. During the continuing inflammation, the
activity of the phagocytic cells is thought to lead to an
inflammatory response in the vessel wall, which in-
volves cellular excretion and proliferation that results

DOI: 10.1097/01.LAB.0000077981.49367.46

Received April 22, 2003.

This study was supported by grants from the Finnish Foundation of Cardiovascular Research, the Elli and Elvi Oksanen Fund of the Pirkanmaa Fund under the auspices of the Finnish Cultural Foundation, Juho Vainio Foundation, the Yrjö Jahnsson Foundation, the Finnish Foundation of Alcohol Studies, the Research Foundation of Orion Corporation, and the Medical Research Fund of the University Hospital of Tampere. Address reprint requests to: Dr. Riikka Mäkelä, Laboratory of Atherosclerosis Genetics, Department of Clinical Chemistry, Center for Laboratory Medicine, University Hospital of Tampere, P.O. Box 2000, 33521 Tampere, Finland. E-mail: riikka.makela@uta.fi in further growth of the lesion (Berliner and Heinecke, 1996; Heinecke, 1998, 1999; Rosenfeld, 1998; Steinberg, 1997).

Myeloperoxidase (MPO) is a heme enzyme found in phagocytes and is considered to be an essential part of the antimicrobial defense system (Klebanoff, 1980; Winterbourn et al, 2000). During the oxidative burst related to phagocyte activation, MPO generates a number of reactive species, such as hypochlorous acid (Harrison and Schultz, 1976), tyrosyl radicals (Heinecke et al, 1993), and reactive nitrogen species (Eiserich et al, 1998; Harrison and Schultz, 1976; Hazen et al, 1996). Reactive intermediates generated by MPO of the coronary wall are thought to constitute an important pathway of oxidative destruction throughout the disease process of atherosclerosis (Heinecke, 1999). MPO is expressed in human atherosclerotic lesions, and stable end-products generated by MPO-derived oxidants are enriched in proteins, lipids, and LDL of the lesions (Daugherty et al, 1994; Hazell et al, 1996; Hazen and Heinecke, 1997; Leeuwenburgh et al, 1997; Malle et al, 2000). Furthermore, in patients with coronary artery disease (CAD) leukocyte- and blood-MPO levels are greater than in controls (Zhang et al, 2001). The protective effect of the MPO deficiency against cardiovascular damage has also been reported (Kutter et al, 2000). 
The promoter region of the MPO gene has a single G-to-A base substitution at position -463 inside a strong SP1 transcription factor consensus sequence. This polymorphism leads to high- (G/G) and lowexpression (A/A, A/G) genotypes in promoter activity and gene expression assays in vitro (Piedrafita et al, 1996). The polymorphism has been previously associated with a susceptibility to several disease states, such as acute promyelocytic leukemia (Reynolds et al, 1997), multiple sclerosis (Nagra et al, 1997), lung cancer (London et al, 1997), and Alzheimer's disease (Reynolds et al, 1999). Whether this genetic variation of the MPO gene is associated with atherosclerosis is not clear. In patients with angiographically documented CAD, the A allele is less frequent (Nikpoor et al, 2001). In contrast it is also known that the A allele is associated with increased lipid levels in the healthy population, suggesting a possible implication of this allele with the risk of CAD (Hoy et al, 2001).

This prompted us to investigate whether the MPO genotypes modify the extent of different types of atherosclerotic lesions in the thoracic and abdominal aorta. The study was performed on an autopsy series of 300 middle-aged Finnish men from the Helsinki Sudden Death Study with autopsy-confirmed atherosclerosis in the abdominal and thoracic aorta (llveskoski et al, 1999).

\section{Results}

\section{Study Population}

The data from autopsies and of MPO genotypes were available in 266 cases, which comprised the final study population. To study the effect of age, the subjects were divided according to median age of 53 years, a cut-off point previously used in the same series (llveskoski et al, 1999). The background characteristics and the categorized MPO genotype frequencies for both age groups are shown in Table 1. Among the 266 autopsies of men aged 33 to 69 years, the MPO genotype frequencies were $180(67 \%)$ for G/G, $79(30 \%)$ for $A / G$, and $7(3 \%)$ for $A / A$. The frequencies of the $G$ and $A$ alleles were 0.81 and 0.19 for men $<53$ years and 0.84 and 0.16 for men $\geq 53$ years, respectively. There were no significant differences in genotype or allele distributions between these two age groups. The distributions of the genotypes followed the Hardy-Weinberg equilibrium. There were no significant differences between genotype or age groups and the characteristics of the study subjects, with the exception of the consumption of alcohol, which was significantly higher among men $<53$ years compared with older men.

The number of cases without atherosclerotic lesions and the proportion of the subjects affected by the atherosclerotic disease are presented in Table 2. For data on the various types of atherosclerotic lesions, see Table 3.

\section{MPO Gene Variation and Atherosclerotic Lesions in Abdominal Aorta}

In analysis of covariance (ANCOVA), age and body mass index (BMI) were used as covariates and the MPO genotype and age group as factors. There were significant MPO genotype-by-age interactions for the percent area of both fibrotic $(p=0.008)$ and calcified $(p=0.015)$ atherosclerotic lesions in the abdominal aorta. Men $<53$ years with the low-expression genotypes $(A / G, A / A)$ had, on average, a $38.6 \%$ larger area of fibrotic lesions $(p=0.017)$ and a $43.8 \%$ larger area of calcified lesions $(p=0.026)$ than did the subjects with the high-expression genotype (G/G) (Table 3). The power of the test measuring the differences between the areas of calcified and fibrotic lesions by MPO genotype was $99 \%$ for fibrotic and $91 \%$ for calcified lesions (124 cases in each group). By linear regression analysis, which included all available atherosclerosis risk factors, MPO genotype turned out to be an independent predictor of fibrotic $(p=0.04)$ and calci-

Table 1. Subjects by MPO Genotype and Age

\begin{tabular}{|c|c|c|c|c|c|c|}
\hline \multirow[b]{2}{*}{ MPO genotype group } & \multicolumn{2}{|c|}{$<53$ Years old } & \multicolumn{2}{|c|}{$\geq 53$ Years old } & \multicolumn{2}{|c|}{ All subjects } \\
\hline & $\mathrm{AA} / \mathrm{AG}$ & $\mathrm{GG}$ & $\mathrm{AA} / \mathrm{AG}$ & GG & $\mathrm{AA} / \mathrm{AG}$ & $\mathrm{GG}$ \\
\hline No. of subjects & 42 & 83 & 44 & 97 & 86 & 180 \\
\hline Age, years ${ }^{a}$ & $42.93 \pm 4.20$ & $43.17 \pm 4.44$ & $60.32 \pm 5.18$ & $59.92 \pm 4.75$ & 51.83 & 52.19 \\
\hline Body mass index, $\mathrm{kg} / \mathrm{m}^{2 a}$ & $24.80 \pm 5.18$ & $24.49 \pm 4.66$ & $24.30 \pm 4.23$ & $25.97 \pm 5.18$ & 24.55 & 25.29 \\
\hline Myocardial infarction (yes/no) & $8 / 34(19 \%)$ & $9 / 74(11 \%)$ & $15 / 29(34 \%)$ & $34 / 63(35 \%)$ & $23 / 63(27 \%)$ & $43 / 137(24 \%)$ \\
\hline Coronary thrombus (yes/no) & $2 / 40(5 \%)$ & $5 / 78(6 \%)$ & $4 / 40(9 \%)$ & $9 / 88(9 \%)$ & $6 / 80(7 \%)$ & $14 / 166(8 \%)$ \\
\hline \multicolumn{7}{|l|}{ Cause of death, $N$} \\
\hline Cardiac disease/other ${ }^{b}$ & $14 / 28(33 \%)$ & $21 / 62(25 \%)$ & $24 / 20(55 \%)$ & $46 / 51(47 \%)$ & $38 / 48(44 \%)$ & $67 / 113(37 \%)$ \\
\hline MP0 genotypes, $N(\%)$ & $42(16 \%)$ & $83(31 \%)$ & $44(17 \%)$ & $97(36 \%)$ & $86(32 \%)$ & $180(68 \%)$ \\
\hline \multicolumn{7}{|l|}{ Coronary risk factor } \\
\hline Diabetes (yes/no) ${ }^{c}$ & $1 / 16(6 \%)$ & $5 / 37(12 \%)$ & $4 / 20(17 \%)$ & $9 / 34(21 \%)$ & $5 / 36(12 \%)$ & $14 / 71(16 \%)$ \\
\hline Hypertension (yes/no) ${ }^{c}$ & $4 / 13(24 \%)$ & $12 / 30(29 \%)$ & 8/16 (33\%) & $15 / 28(35 \%)$ & $12 / 29(29 \%)$ & $27 / 58(32 \%)$ \\
\hline Smoking (yes/no) ${ }^{d}$ & $13 / 9(59 \%)$ & $34 / 7(83 \%)$ & $18 / 12(60 \%)$ & $34 / 16(68 \%)$ & $31 / 21(60 \%)$ & $68 / 23(75 \%)$ \\
\hline Alcohol $\mathrm{g} / \mathrm{d}^{a c}$ & $158.3 \pm 121.0$ & $118.5 \pm 101.5$ & $96.7 \pm 124.4$ & $70.3 \pm 82.5$ & $124.8 \pm 125.4$ & $92.2 \pm 94.2$ \\
\hline
\end{tabular}

MPO, myeloperoxidase.

${ }^{a}$ Values are mean \pm standard deviation.

${ }^{b}$ Includes causes of death due to other diseases, violence, intoxication, and unknown reasons $(N=6)$.

${ }^{c}$ Data available in 126 cases.

${ }^{d}$ Data available in 143 cases; ex-smokers are included in the group of nonsmokers. 
Table 2. The Number and Proportion of Cases with or Without Different Atherosclerotic Lesion Types in Abdominal and Thoracic Aorta

\begin{tabular}{|c|c|c|c|c|c|c|c|c|c|}
\hline & \multicolumn{3}{|c|}{$<53$ Years old $(N=125)$} & \multicolumn{3}{|c|}{$\geq 53$ Years old $(N=141)$} & \multicolumn{3}{|c|}{ All $(N=266)$} \\
\hline & \multicolumn{2}{|c|}{ Lesions } & \multirow[b]{2}{*}{ Affected, \% } & \multicolumn{2}{|c|}{ Lesions } & \multirow[b]{2}{*}{ Affected, \% } & \multicolumn{2}{|c|}{ Lesions } & \multirow[b]{2}{*}{ Affected, \% } \\
\hline & No & Yes & & No & Yes & & No & Yes & \\
\hline \multicolumn{10}{|c|}{ Abdominal aorta } \\
\hline Fatty streak & 0 & 125 & 100 & 0 & 141 & 100 & 0 & 266 & 100 \\
\hline Fibrotic & 20 & 105 & 84 & 1 & 140 & 99.3 & 21 & 239 & 89.8 \\
\hline Calcified & 42 & 83 & 66.4 & 3 & 138 & 97.9 & 45 & 221 & 83.1 \\
\hline \multicolumn{10}{|l|}{ Thoracic aorta } \\
\hline Fatty streak & 8 & 117 & 93.6 & 1 & 140 & 99.3 & 9 & 257 & 96.6 \\
\hline Fibrotic & 60 & 65 & 52 & 11 & 130 & 92.2 & 71 & 195 & 73.3 \\
\hline Calcified & 96 & 29 & 23.2 & 27 & 114 & 80.9 & 123 & 143 & 53.8 \\
\hline
\end{tabular}

fied $(p=0.001)$ lesion area in men $<53$ years (Table 4). No statistically significant associations for MPO genotype were found among men $\geq 53$ years. For the other types of lesions in abdominal aorta, there were no significant differences between the study groups (Table 3).

In ANCOVA involving all of the known risk factors as covariates, the interaction remained significant in fibrotic $(p=0.038)$ and in calcified $(p=0.053)$ lesion areas in abdominal aorta. When the possible confounding factor, alcohol consumption, was added to the linear regression analysis as an independent variable, MPO genotype remained as an explanatory variable in fibrotic $(p=0.055)$ and calcified $(p=0.001)$ lesions in abdominal aorta among men $<53$ years; however, the entire regression model was not significant in fibrotic lesions.

\section{MPO Gene Variation and Atherosclerotic Lesions in Thoracic Aorta}

By ANCOVA, there was a statistically significant $p=$ 0.003) interaction between MPO genotype and age group regarding the fibrotic lesion area in the thoracic aorta when age and BMI were used as covariates. Men $\geq 53$ years with high-expression genotype (G/G) had on average a $24.5 \%$ larger area of fibrotic lesions $(p=0.012)$ than subjects with low-expression genotypes (A/G, A/A) (Table 3). The power of the test to measure the difference between the area of fibrotic lesions according to MPO genotypes was $80 \%$ with 136 cases. In addition, MPO genotype did not remain as an independent predictor of fibrotic lesions when analyzed by linear regression adjusted for the available atherosclerosis risk factors. There were no associations found among men $<53$ years, and there were no significant differences between the age-genotype groups as the other types of lesions were concerned (Table 3).

For the statistically significant areas of atherosclerotic lesions in abdominal and thoracic aortas, the one-way ANOVA (ANOVA) was performed broken down by age quartiles and using the MPO genotype as a factor (Table 5).

\section{Discussion}

To our knowledge, no previous studies have investigated the association between MPO gene promoter polymorphism and the severity of atherosclerosis using arterial wall samples obtained at autopsies. We found an age-dependent association between MPO gene promoter $-463 \mathrm{G} / \mathrm{A}$ genotype and the percent area of fibrotic and calcified atherosclerotic lesions in the aorta. The MPO low-expression genotypes (A/A and $A / G$ ) were associated with larger fibrotic and calcified lesions in men $<53$ years in both ANCOVA and linear regression analysis, but the effect was abrogated as the age exceeded 53 years. Men $\geq 53$ years carrying the MPO high-expression genotype $(G / G)$ had larger areas of fibrotic lesions, especially in the thoracic aorta and to a lesser degree also in the abdominal aorta. However, this association is suggestive, and the MPO genotype did not appear as an independent predictor in linear regression analysis among men $\geq 53$ years.

The proportions of the MPO genotypes in our study population were comparable to what has previously been published concerning the general Caucasian population (Reynolds et al, 1997). In earlier reports MPO -463G/A polymorphism has been studied in several disease states. The A allele has been reported to have a protective role in the occurrence of symptomatic and angiographically proved CAD, but the stage of possible asymptomatic CAD of the controls was not evaluated in the study (Nikpoor et al, 2001). The G/G genotype is claimed to be a risk factor in lung cancer (London et al, 1997), but in Finnish men no direct association has been found (Misra et al, 2001). In addition, no difference in serum MPO levels between the genotypes has been detected (Hoy et al, 2001). Our results imply that the high-expression genotype G/G offers protection from the development of atherosclerosis. In line with our observation, a recent report found that subjects carrying the $A$ allele displayed higher levels of atherosclerosis risk factors, triglycerides, total cholesterol, LDL cholesterol, and apolipoprotein B than subjects with the G allele. (Hoy et al, 2001). Owing to the sudden and unexpected 


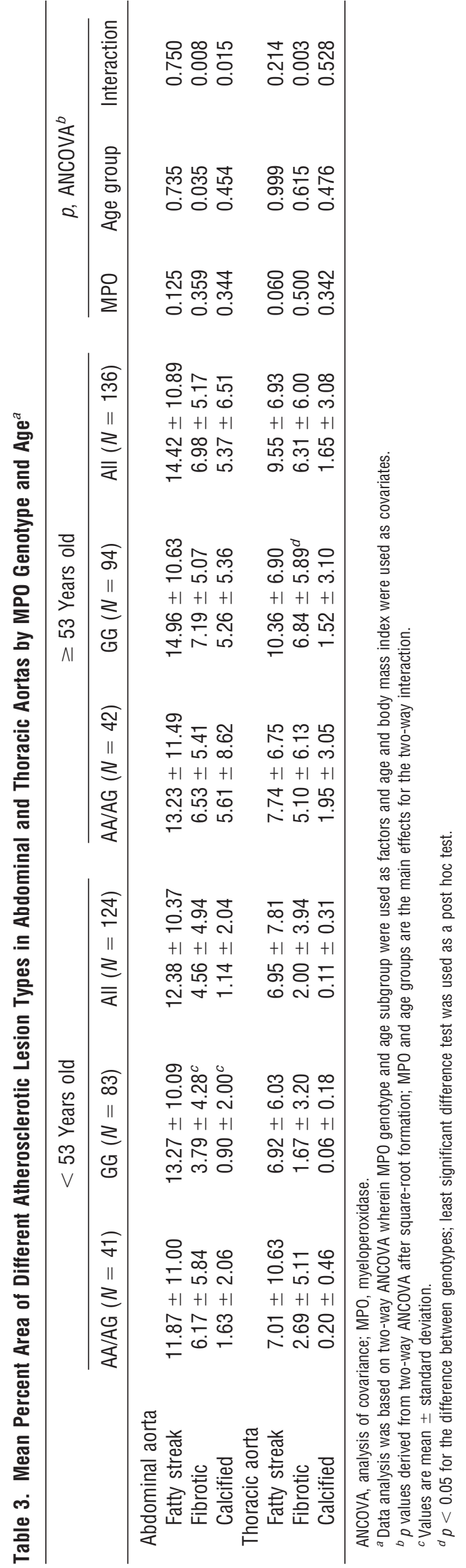

nature of death, the possible confounding effect of serum cholesterol or other lipid levels in the deceased were not available and could not been taken into account in statistical analysis.

The effect of the MPO polymorphism on atherosclerosis development was age dependent, and the interaction was stronger in men $<53$ years. This finding is somewhat in agreement with the conclusions of the Swedish twin study (Marenberg et al, 1994), wherein genetic factors were suggested to play a greater role in death due to coronary heart disease at a younger age, and at an older age, the effect of environmental or acquired factors tended to dampen the inherited dispositions. It is also possible that our results are due to linkage disequilibrium of the $-463 \mathrm{G} / \mathrm{A}$ polymorphism, with some other functional MPO polymorphism being responsible for more advanced atherosclerosis.

There were significant interactions of MPO genotype with lesions with fibrous tissue and calcification. According to the inflammation theory of atherosclerosis development, the presence of phagocytic cells in the developing atheroma is thought to contribute to the continuing accumulation of oxidized LDL; this leads to increased matrix synthesis in fibrotic lesions and then to a calcification process (Berliner et al, 1995; Berliner and Heinecke, 1996; Lusis, 2000; Ross, 1999). MPO and proteins modified by MPO have been detected from advanced human atherosclerotic plaques, whereas only a small amount of MPO is present in human coronary artery fatty streaks (Daugherty et al, 1994; Heinecke, 1998; Malle et al, 2000; Sugiyama et al, 2001). This is congruent with our study, in which no significant differences were found between the genotypes in the mean area of fatty streaks.

In summary, the results of our study suggest that there is an interaction for the genetic variant of the MPO gene with the progression of atherosclerosis, similar to that previously found for apolipoprotein $\mathrm{E}$ epsilon 4 (Ilveskoski et al, 1999). The precise role of MPO and its interaction with other genetic and acquired factors involved in the development of atherosclerosis require more investigation.

\section{Materials and Methods}

\section{Subjects}

The Helsinki Sudden Death Study was designed to study the acquired and inherited risk factors predisposing to sudden death in Finnish middle-aged men. An autopsy series including 300 consecutive Caucasian men aged 33 to 69 years was collected during 12 months from 1991 to 1992 at the Department of Forensic Medicine, University of Helsinki, as previously described (llveskoski et al, 1999; Mikkelsson et al, 2000). The cause of death was cardiac disease in $39 \%(n=105)$, other diseases in $21 \%(n=55)$, and violent death (suicides and accidents) in $40 \%(n=$ 106) (Table 1). 
Table 4. Strongest Predictors of the Area of Fibrotic and Calcified Atherosclerotic Lesions in Abdominal Aorta ${ }^{a}$

\begin{tabular}{|c|c|c|c|c|c|}
\hline \multirow[b]{2}{*}{ Age group } & \multirow[b]{2}{*}{ Explanatory variables (independent) } & \multicolumn{2}{|c|}{ Fibrotic lesions } & \multicolumn{2}{|c|}{ Calcified lesions } \\
\hline & & Beta $^{b}$ & $p$ Value & Beta $^{b}$ & $p$ Value \\
\hline \multirow[t]{6}{*}{$<53$ Years } & MPO genotype & 0.265 & 0.037 & 0.398 & 0.001 \\
\hline & $\mathrm{BMI}$ & -0.021 & 0.877 & -0.110 & 0.347 \\
\hline & Age & 0.320 & 0.015 & 0.456 & 0.000 \\
\hline & Hypertension (yes or no) & 0.213 & 0.102 & 0.069 & 0.539 \\
\hline & Diabetes (yes or no) & 0.095 & 0.454 & 0.185 & 0.100 \\
\hline & Entire model $\left(\mathrm{R}^{2}\right)$ & 0.231 & 0.017 & 0.324 & $<0.001$ \\
\hline \multirow[t]{6}{*}{$\geq 53$ Years } & MPO genotype & -0.118 & 0.378 & -0.011 & 0.933 \\
\hline & $\mathrm{BMI}$ & -0.033 & 0.817 & -0.313 & 0.023 \\
\hline & Age & 0.182 & 0.172 & 0.185 & 0.143 \\
\hline & Hypertension (yes or no) & -0.067 & 0.632 & 0.109 & 0.414 \\
\hline & Diabetes (yes or no) & -0.055 & 0.671 & -0.016 & 0.897 \\
\hline & Entire model $\left(\mathrm{R}^{2}\right)$ & 0.053 & 0.667 & 0.054 & 0.146 \\
\hline
\end{tabular}

BMI, body mass index; MPO, myeloperoxidase.

${ }^{a}$ Linear regression analysis was carried out in both age groups to identify the independent risk factors for atherosclerotic lesions.

${ }^{b}$ Standardized coefficients.

\section{Measuring the Area of Atherosclerotic Lesions by Computer-Assisted Morphometry}

The definition of atherosclerosis was based on the protocols of two international studies: the International Atherosclerosis Project, Standard Operating Protocol (Guzman et al, 1968); and the WHO Study Group in Europe (Uemura et al, 1964). Whole-mounted proximal parts of the abdominal and thoracic aorta were collected for analysis. The area involved with fatty streaks, fibrotic plaques, and calcified lesions was measured by computer-assisted planimetry, which is a standard planimetric machine connected to a personal computer. The procedure of the atherosclerotic lesion measurements has been described by Ilveskoski et al (1999).

\section{Risk Factors for Atherosclerosis Disease}

A spouse, relative, or close friend of the deceased was reached and interviewed in 147 cases. Among the questions pertaining to the risk factors for sudden death (ie, arterial hypertension and diabetes), there were questions to establish past and recent smoking and drinking habits of the deceased. Complete data on all risk factors in addition to MPO genotype and autopsy data were available in 126 cases, which comprised the adjusted study population.

\section{Determination of the MPO Genotype}

DNA was isolated from frozen cardiac muscle samples by a standard phenol extraction method. A region surrounding the $-463 \mathrm{G} / \mathrm{A}$ variant of the MPO gene was amplified using PCR, and the primary and secondary round primers were designed as previously described by London and colleagues (London et al, 1997). After digestion of the PCR product with Acil restriction endonuclease (New England Biolabs Inc., Beverly, Massachusetts), fragments were separated using agarose gel (2\%) electrophoresis and were visualized by ethidium bromide staining.

\section{Statistical Analyses}

Data analysis was based on two-way ANCOVA and linear regression. Non-normally distributed data were square-root transformed prior to analysis, but the results are displayed as the crude values. To achieve a suitable group size, MPO genotypes were categorized into high-expression (G/G) and low-expression (A/G, A/A) allele groups, a categorization previously used in another study (Reynolds et al, 2000). To adjust for possible differences between younger persons whose atherosclerosis process was not far advanced and older persons with a longer disease period, the data were divided into two age groups, $<53$ and $\geq 53$ years old by the median age (53 years). The MPO genotype and age subgroups were used as factors in the two-way ANCOVA, in which the possible confounding effects of age and BMl were taken into account by including them into the model as covariates. The least significant difference test was used as a post hoc test to study the differences between the genotype groups. We also used linear regression analysis to identify independent risk factors for atherosclerotic lesions in both age groups. The analyses were carried out separately in both age subgroups. The mean areas of fibrotic and calcified lesions were included as independent variables in two separate models in which MPO genotype group, age, BMI, hypertension (yes/no), diabetes (yes/no), and smoking (yes/no) were included as explanatory variables. Oneway ANOVA was used for the statistical analysis broken down by the age quartiles, and MPO genotype was used as a factor for the analysis. The statistical analyses were performed using Statistica for Windows 5.1 (StatSoft Inc., Tulsa, Oklahoma), and SPSS 9.0 for Windows 95 software (SPSS Inc., Chicago, Illinois). The power $(1-\beta)$ of the test procedures was calculated 


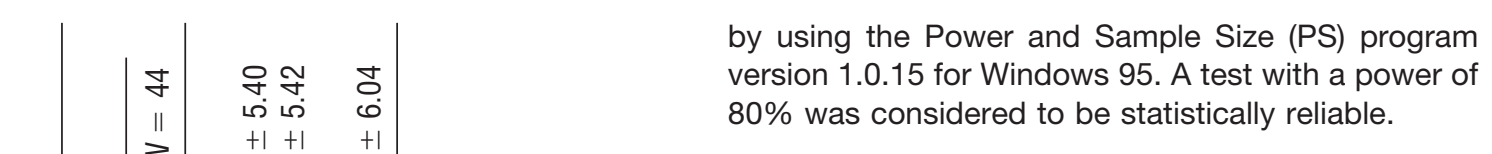

Acknowledgements

The authors thank Seppo Tyynelä for the planimetric measurements, Anna-Maija Koivisto for the statistical guidance, and Marita Koli for her skillful technical assistance.

\section{References}

Babior BM (2000). Phagocytes and oxidative stress. Am J Med 109:33-44.

Berliner JA and Heinecke JW (1996). The role of oxidized lipoproteins in atherogenesis. Free Radic Biol Med 20:707727.

Berliner JA, Navab M, Fogelman AM, Frank JS, Demer LL, Edwards PA, Watson AD, and Lusis AJ (1995). Atherosclerosis: Basic mechanisms. Oxidation, inflammation, and genetics. Circulation 91:2488-2496.

Daugherty A, Dunn JL, Rateri DL, and Heinecke JW (1994). Myeloperoxidase, a catalyst for lipoprotein oxidation, is expressed in human atherosclerotic lesions. J Clin Invest 94: 437-444.

Eiserich JP, Hristova M, Cross CE, Jones AD, Freeman BA, Halliwell B, and van der Vliet A (1998). Formation of nitric oxide-derived inflammatory oxidants by myeloperoxidase in neutrophils. Nature 391:393-397.

Guzman MA, McMahan CA, McGill HC, Strong JP, Tejada C, Restrepo C, Eggen DA, Robertson WB, and Solberg LA (1968). Selected methodologic aspects of the International Atherosclerosis Project. Lab Invest 18:479-497.

Harrison JE and Schultz J (1976). Studies on the chlorinating activity of myeloperoxidase. J Biol Chem 251:1371-1374.

Hazell LJ, Arnold L, Flowers D, Waeg G, Malle E, and Stocker $R$ (1996). Presence of hypochlorite-modified proteins in human atherosclerotic lesions. J Clin Invest 97:1535-1544.

Hazen SL and Heinecke JW (1997). 3-Chlorotyrosine, a specific marker of myeloperoxidase-catalyzed oxidation, is markedly elevated in low density lipoprotein isolated from human atherosclerotic intima. J Clin Invest 99:2075-2081.

Hazen SL, Hsu FF, Mueller DM, Crowley JR, and Heinecke JW (1996). Human neutrophils employ chlorine gas as an oxidant during phagocytosis. J Clin Invest 98:1283-1289.

Heinecke JW (1998). Oxidants and antioxidants in the pathogenesis of atherosclerosis: Implications for the oxidized low density lipoprotein hypothesis. Atherosclerosis 141:1-15.

Heinecke JW (1999). Mechanisms of oxidative damage by myeloperoxidase in atherosclerosis and other inflammatory disorders. J Lab Clin Med 133:321-325.

Heinecke JW, Li W, Daehnke HL, and Goldstein JA (1993). Dityrosine, a specific marker of oxidation, is synthesized by the myeloperoxidase-hydrogen peroxide system of human neutrophils and macrophages. J Biol Chem 268:4069-4077.

Hoy A, Tregouet D, Leininger-Muller B, Poirier O, Maurice M, Sass C, Siest G, Tiret L, and Visvikis S (2001). Serum myeloperoxidase concentration in a healthy population: Biological variations, familial resemblance and new genetic polymorphisms. Eur J Hum Genet 9:780-786. 
Ilveskoski E, Perola M, Lehtimaki T, Laippala P, Savolainen V, Pajarinen J, Penttila A, Lalu KH, Mannikko A, Liesto KK, Koivula T, and Karhunen PJ (1999). Age-dependent association of apolipoprotein $\mathrm{E}$ genotype with coronary and aortic atherosclerosis in middle-aged men: An autopsy study. Circulation 100:608-613.

Klebanoff SJ (1980). Oxygen metabolism and the toxic properties of phagocytes. Ann Intern Med 93:480-489.

Kutter D, Devaquet P, Vanderstocken G, Paulus JM, Marchal $V$, and Gothot A (2000). Consequences of total and subtotal myeloperoxidase deficiency: Risk or benefit? Acta Haematol 104:10-15.

Leeuwenburgh C, Rasmussen JE, Hsu FF, Mueller DM, Pennathur S, and Heinecke JW (1997). Mass spectrometric quantification of markers for protein oxidation by tyrosyl radical, copper, and hydroxyl radical in low density lipoprotein isolated from human atherosclerotic plaques. $J$ Biol Chem 272:3520-3526.

London SJ, Lehman TA, and Taylor JA (1997). Myeloperoxidase genetic polymorphism and lung cancer risk. Cancer Res 57:5001-5003.

Lusis AJ (2000). Atherosclerosis. Nature 407:233-241.

Malle E, Waeg G, Schreiber R, Grone EF, Sattler W, and Grone HJ (2000). Immunohistochemical evidence for the myeloperoxidase/H2O2/halide system in human atherosclerotic lesions. Eur J Biochem 267:4495-4503.

Marenberg ME, Risch N, Berkman LF, Floderus B, and de Faire U (1994). Genetic susceptibility to death from coronary heart disease in a study of twins. N Engl J Med 330:10411046.

Mikkelsson J, Perola M, Wartiovaara U, Peltonen L, Palotie A, Penttila A, and Karhunen PJ (2000). Plasminogen activator inhibitor-1 (PAl-1) 4G/5G polymorphism, coronary thrombosis, and myocardial infarction in middle-aged Finnish men who died suddenly. Thromb Haemost 84:78-82.

Misra RR, Tangrea JA, Virtamo J, Ratnasinghe D, Andersen MR, Barrett M, Taylor PR, and Albanes D (2001). Variation in the promoter region of the myeloperoxidase gene is not directly related to lung cancer risk among male smokers in Finland. Cancer Lett 164:161-167.

Nagra RM, Becher B, Tourtellotte WW, Antel JP, Gold D, Paladino T, Smith RA, Nelson JR, and Reynolds WF (1997). Immunohistochemical and genetic evidence of myeloperoxidase involvement in multiple sclerosis. J Neuroimmunol 78:97-107.
Nikpoor B, Turecki G, Fournier C, Théroux $P$, and Rouleau GA (2001). A functional myeloperoxidase polymorphic variant is associated with coronary artery disease in FrenchCanadians. Am Heart J 142:336-339.

Piedrafita FJ, Molander RB, Vansant G, Orlova EA, Pfahl M, and Reynolds WF (1996). An Alu element in the myeloperoxidase promoter contains a composite SP1-thyroid hormoneretinoic acid response element. J Biol Chem 271:1441214420.

Reynolds WF, Chang E, Douer D, Ball ED, and Kanda V (1997). An allelic association implicates myeloperoxidase in the etiology of acute promyelocytic leukemia. Blood 90: 2730-2737.

Reynolds WF, Hiltunen M, Pirskanen M, Mannermaa A, Helisalmi S, Lehtovirta M, Alafuzoff I, and Soininen H (2000). MPO and APOEepsilon4 polymorphisms interact to increase risk for AD in Finnish males. Neurology 55:1284-1290.

Reynolds WF, Rhees J, Maciejewski D, Paladino T, Sieburg H, Maki RA, and Masliah E (1999). Myeloperoxidase polymorphism is associated with gender specific risk for Alzheimer's disease. Exp Neurol 155:31-41.

Rosenfeld ME (1998). Inflammation, lipids, and free radicals: Lessons learned from the atherogenic process. Semin Reprod Endocrinol 16:249-261.

Ross R (1999). Atherosclerosis: An inflammatory disease. N Engl J Med 340:115-126.

Steinberg D (1997). Low density lipoprotein oxidation and its pathobiological significance. J Biol Chem 272:20963-20966.

Sugiyama S, Okada Y, Sukhova GK, Virmani R, Heinecke JW, and Libby $P$ (2001). Macrophage myeloperoxidase regulation by granulocyte macrophage colony-stimulating factor in human atherosclerosis and implications in acute coronary syndromes. Am J Pathol 158:879-891.

Uemura K, Sternby N, Vanecek R, Vihert A, and Kagan A (1964). Grading atherosclerosis in aorta and coronary arteries obtained at autopsy: Application of a tested method. Bull World Health Org 31:297-320.

Winterbourn CC, Vissers MC, and Kettle AJ (2000). Myeloperoxidase. Curr Opin Hematol 7:53-58.

Zhang R, Brennan ML, Fu X, Aviles RJ, Pearce GL, Penn MS, Topol EJ, Sprecher DL, and Hazen SL (2001). Association between myeloperoxidase levels and risk of coronary artery disease. JAMA 286:2136-2142. 